\title{
Evaluación del efecto antagónico de una biopelícula con extractos de Samanea saman frente a Colletotrichum gloeosporioides responsable de la antracnosis en mango

\author{
Evaluation of the antagonistic effect of a biofilm with extracts of Samanea saman against \\ Colletotrichum gloeosporioides responsible for anthracnose in mango
}

Miguel Moreno Paredes ${ }^{*}$, Lenín Ramiréz-Cando ${ }^{1,2}$, Elisa Cedeño Luzardo ${ }^{3}$

DOI. 10.21931/RB/2021.06.01.5

Resumen: La actividad antagónica de extractos etanòlicos (EE) e hidroetanólicos (EHE) de corteza y metanólicos (EM) de fruto de Samanea saman fue evaluada in vitro mediante el método de difusión en pozo frente al hongo patógeno Colletotrichum gloesporioides; responsable de la antracnosis, con el fin de evaluar su idoneidad para incorporarlos en recubrimientos biodegradables que ayuden en el control de enfermedades postcosecha en mango (Mangifera indica). Solo el EM mostro actividad inhibitoria significativa (CMI $140 \mu \mathrm{L} / \mathrm{mL})$ en crudo, con un IC50 de $81,85 \mu \mathrm{L} / \mathrm{mL}$. Al aumentar la concentración del EM en el sistema recubrimiento-extracto de $140 \mu \mathrm{L} / \mathrm{mL}$ a $200 \mu \mathrm{L} / \mathrm{mL}$ se consiguió una respuesta inhibitoria considerablemente mayor, Llevando el crecimiento micelial a niveles de reproducción bajo para el hongo en estudio. El tamizaje fitoquímico de los diferentes solventes mostró la presencia de metabolitos secundarios de manera variada; sin embargo, comparativamente el extracto metanólico (EM) del fruto presento la mayor cantidad de estos compuestos tales como alcaloides, taninos, saponinas, terpenoides y resinas que han sido identificados por sus propiedades, como potentes antimicrobianos y antioxidantes naturales

Palabras clave: Antracnosis, biopelícula, Colletotrichum gloesporioides, difusión en pozo, Samanea saman, tamizaje fitoquímico.

Abstract: The antagonistic activity of ethanolic (EE) and hydroethanolic (EHE) extracts from bark and methanolic (EM) from Samanea saman fruit was evaluated in vitro using the suitable diffusion method against the pathogenic fungus Colletotrichum gloesporioides; responsible for anthracnose, to evaluate their suitability for incorporation in biodegradable coatings that help control post-harvest diseases in mango (Mangifera indica). Only EM showed significant inhibitory activity (MIC140 $\mu \mathrm{L} / \mathrm{mL}$ ) in crude oil, with an IC50 of $81.85 \mu \mathrm{L} / \mathrm{mL}$. By increasing the concentration of EM in the coating-extract system from $140 \mu \mathrm{L} / \mathrm{mL}$ to $200 \mu \mathrm{L} / \mathrm{mL}$, a considerably higher inhibitory response was achieved, bringing mycelial growth to low reproduction levels the fungus under study. The phytochemical screening of the different solvents showed the presence of secondary metabolites in a varied way; However, comparatively, the methanolic extract (EM) of the fruit presented the highest amount of these compounds such as alkaloids, tannins, saponins, terpenoids, and resins that have been identified for their properties, as potent antimicrobials and natural antioxidants.

Key words: Anthracnose, biofilm, Colletotrichum gloesporioides, well diffusion, Samanea saman, phytochemical screening.

\section{Introducción}

La antracnosis es la principal enfermedad postcosecha que afecta al mango a nivel mundial, su incidencia puede alcanzar casi el cien por ciento del total de fruta cosechada en zonas de alta humedad y fuertes precipitaciones ${ }^{1}$; siendo el hongo Colletotrichum y su teleomorfo los patógenos más extendidos a nivel mundial, causantes de esta enfermedad². La antracnosis se ha posicionado como una de las principales causas por las cuales el mango ecuatoriano no puede ubicarse con ventaja en mercados internacionales frente a sus competidores directos ${ }^{3}$. Por este motivo, en la actualidad se ha desarrollado el uso e implementación de recubrimientos biodegradables o "biopelículas" qué; junto con inhibir reacciones metabólicas asociadas con la maduración y mantener las propiedades mecánicas y sensoriales, pueden actuar en sinergia con agentes antimicrobianos y antioxidantes 4,5 .

El Samanea saman es considerado como un árbol multipropósito; ya que no solo posee gran potencialidad como alimento alternativo para rumiantes y monogástricos en épocas de sequía, sino también por ser fuente importante de metabo- litos secundarios que demuestran sinergismo y alto potencial antimicrobiano ${ }^{6}$; siendo la corteza y los frutos de este, identificados como las principales fuentes de estos metabolitos, específicamente de taninos condensados y alcaloides?.

Por las razones arriba expuestas, se hace imperativo el brindar alternativas innovadoras que ayuden al sector agroindustrial, y al desarrollo de su capacidad exportable; brindando al mercado internacional, productos de alta calidad organoléptica y de inocuidad alimentaria asegurada.

Estudios recientes han reportado que existen alrededor de 2400 especies vegetales con propiedades biocída; y de estos, 400 especies presentan propiedad fungicida contra 142 hongos diferentes. Los compuestos antimicrobianos pueden estar presentes en diferentes órganos de la planta, por ejemplo, en semillas, flores, tallos, raíces y hojas y su actividad depende de la concentración, método de extracción, edad de la planta y época de cosecha, entre otros ${ }^{8}$.

Diversos estudios han identificado a los compuestos activos responsables de estas propiedades antimicrobianas tales 
como fenoles, taninos, alcaloides, saponinas, terpenoides, cumarinas, glicosidos, entre otros ${ }^{8}$.

El Samanea samán además de su utilidad como alimento animal, tiene otros usos importantes como planta melífera, maderable y medicinal; y es justamente en este último aspecto, que resulta de mucho interés en la actualidad por ser una planta con actividad antimicrobiana debido a los compuestos secundarios presentes en ella9.

De manera empírica, las hojas, la corteza, la raíz, las semillas y las vainas del Samanea saman han sido usados en el sistema de medicina tradicional. El extracto alcohólico de las hojas inhibe al Mycobacterium tuberculosis y su infusión es usada como laxante, las semillas se mastican para tratar el dolor de garganta y la decocción de la corteza interna y las hojas frescas se usan para el tratamiento de la diarrea, los resfriados y enfermedades intestinales ${ }^{10}$

La corteza, follaje y frutos secos tienen la más baja humedad; por lo que pueden ser conservados por largos periodos, volviéndose importantes desde el punto de vista investigativo. El Tamizaje fitoquímico realizado evidencio que en la corteza y los frutos aparecen identificados los mayores números de metabolitos secundarios; siendo los más abundantes alcaloides, taninos, carbohidratos, saponinas, aminoácidos, azucares reductores y flavonoides ${ }^{7}$.

Tamizajes fitoquímicos efectuados a las vainas del Samanea saman revelan la presencia de metabolitos secundarios tales como saponinas, esteroides, alcaloides, taninos, flavonoides y resinas. Análisis cualitativos y cuantitativos encontraron que los taninos eran del tipo condensados y tienen un gran potencial antimicrobiano y anti fúngico ${ }^{6}$

Los taninos han sido reportados por prevenir el desarrollo de microorganismos a través de la precipitación de la proteína microbiana y volviéndolas no disponibles para su metabolismo ${ }^{11}$.

Ensayos de cloruro férrico a la corteza del Samanea saman han encontrado la presencia de abundantes taninos del tipo pirocatecolicos; además, en extractos más polares se detectaron azucares, flavonoides, quinonas, y resinas ${ }^{7}$.

Evaluaciones del proceso de extracción de metabolitos secundarios del S. saman con diferentes solventes encontraron que el etanol brinda mejores características de recuperación para la corteza ${ }^{12}$.

Análisis al proceso de extracción de metabolitos secundarios del S. saman con diferentes solventes encontraron que el metanol brinda mejores características de recuperación para el fruto ${ }^{13}$

El uso de recubrimientos de frutas con biopelículas es una tendencia tecnológica que disminuye la perdida de humedad, compuestos volátiles, disminución de la tasa de respiración y retarda la oxidación enzimática. Recubrimientos comestibles a partir de almidones obtenidos de fuentes como arroz o yuca han sido aplicados en toronja, piña, guayaba, pera y mango.

En mango, recubrimientos formulados con galactomananos no retrasan el ablandamiento y reducen la pérdida de peso, la actividad enzimática y la peroxidación lipídica ${ }^{14}$.

Las películas y recubrimientos comestibles están formados por tres componentes principales: polímero, solvente y plastificante. La incorporación de agentes antimicrobianos a biopelículas tiene como objetivo el extender la vida de anaquel de un producto y reducir el crecimiento de patógenos en la superficie de los alimentos.

Los diferentes agentes antimicrobianos que son incorporados a películas o recubrimientos comestibles por sus diversas características químicas forman parte de la estructura de la película mediante la interacción con el polímero y el plas- tificante impidiendo que el compuesto antimicrobiano migre. Sin embargo, el avance de las técnicas analíticas ha puesto en duda la inocuidad de algunas sustancias antimicrobianas que se consideraban seguras; por lo cual, la tendencia actual se ha centrado en la búsqueda de agentes antimicrobianos de origen natural 4 .

La antracnosis es considerada la principal enfermedad fúngica que afecta al mango. Los hongos filamentosos del género Colletotrichum y su teleomorfo son considerados los patógenos de plantas más ampliamente distribuidos a nivel mundial. La variabilidad en la patogenicidad mostró que todas las especies de mangos son susceptibles al microorganismo.

La incidencia de antracnosis es de casi el cien por ciento en fruta producida en climas con alta humedad y fuertes precipitaciones ${ }^{15}$

La más baja y segura temperatura a la que pueden ser expuestos los mangos verdes maduros por largo tiempo es de $120 \mathrm{C}^{16}$.

La temperatura optima de crecimiento para el hongo Colletotrichum gloeosporioides es de $24 \pm 2 \mathrm{oC}^{2}$.

La Food and Drug Administration (FDA) ha aprobado al método de "Difusión por Disco o en Pozo" (Disc-difussion method) como la técnica más usada para el análisis de actividad antimicrobiana de extractos y aceites esenciales en estudios preliminares $^{17}$

La técnica con sensidiscos presenta algunas desventajas; principalmente causadas por la composición del papel filtro "Whatman", que puede intervenir con algunos compuestos catiónicos de los productos naturales evaluados impidiendo su difusión en el agar, este fenómeno es eliminado cuando el método se utiliza directamente en pozo ${ }^{18}$.

La efectividad del efecto antimicótico de diferentes extractos vegetales y sus constituyentes han sido calculados a través del porcentaje de inhibición del crecimiento micelial ${ }^{19}$.

Este trabajo tiene como objetivo evaluar el efecto antagónico frente al hongo "Colletotrichum gloeosporioides"; que provoca antracnosis en mango (Mangifera indica) mediante la aplicación de una biopelícula con extractos de corteza y fruto de "Samanea samán" a través de la identificación de los métodos de extracción más idóneos para la mejor recuperación de metabolitos secundarios en los extractos de las matrices de corteza y fruto de "Samanea samán" mediante un tamizaje fotoquímico cualitativo. Posteriormente establecer las dosis mínimas inhibitorias; de entre los extractos con mejores perfiles de metabolitos secundarios de las matrices en estudio, frente a una cepa certificada de "Colletotrichum gloeosporioides" mediante un análisis in vitro CMI (Concentración Mínima Inhibitoria).

Y finalmente evaluar el efecto antagónico de soluciones formuladas de biopelícula/extractos de las matrices corteza y fruta de "Samanea samán" frente a una cepa certificada del hongo "Colletotrichum gloeosporioides" que produce antracnosis en mango (Mangifera indica).

Validando la hipótesis que la biopelícula con extractos de corteza y/o fruto de Samanea samán tienen efecto antagónico frente al hongo Colletotrichum gloeosporioides que provoca antracnosis en mango (Mangífera indica).

\section{Materiales y métodos}

Las muestras de corteza y fruto de Samanea samán fueron obtenidas de árboles maduros y visiblemente sanos del área recreativa "Sendero del Parque Samanes" ubicado en la parroquia Tarqui; en el sector norte de la ciudad de Guayaquil, 
delimitados al norte por la Av. Casuarina, al Sur Av. S/N, al este por el rio Daule y al oeste por la Vía a Daule con coordenadas

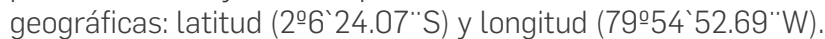

Mediante tres procedimientos se realizó la obtención de los extractos de las diferentes matrices del Samanea samán que fueron: extracto etanólico, hidro etanólico de corteza y metanólico de frutos tomando como referencia la metodología usada por Obasi Nnamdi et al.13.

Para el tratamiento de las matrices se usó como referencia la técnica presentada por Aguilar J. y Col. ${ }^{12}$ procediendo de la siguiente manera:

Las muestras de corteza y fruto obtenidas de árboles maduros y visiblemente sanos se limpiaron manualmente para eliminar restos de materiales extraños luego se secaron al sol por un máximo de 72 horas con el fin de reducir la humedad superficial de las mismas. Posteriormente estas muestras fueron secadas en estufa por un máximo de 72 horas a una temperatura promedio de $60^{\circ} \mathrm{C}$. Las muestras secas se molieron usando un molino de bolas hasta alcanzar un tamaño de partícula de $8 \mu \mathrm{m}$ validados por un tamiz \#8 y se empacaron dos muestras molida de $60 \mathrm{~g}$ de corteza en dos cartuchos de papel filtro whatman \# 54 (tamaño de poro: $22 \mathrm{~mm}$ ) y colocadas cada una en un soxhlet de $300 \mathrm{~mL}$ y $60 \mathrm{~g}$ de muestra molida de fruto se empacaron en un cartucho de papel filtro whatman \# 54 (tamaño de poro: $22 \mathrm{~mm}$ ) y colocadas en un soxhlet de $300 \mathrm{~mL}$

\section{Extracto Etanólico de Corteza de $S$. samán}

Al cartucho que contiene la muestra molida se le adicionó $200 \mathrm{~mL}$ de etanol al $96 \%$ de pureza y se puso en operación el sistema soxhlet a reflujo por 2 horas a $70^{\circ} \mathrm{C}$

\section{Extracto Hidro-Etanólico de Corteza de S. samán}

Al cartucho que contiene la muestra molida se le adicionó $200 \mathrm{~mL}$ de una solución etanol al 96\% de pureza y agua destilada en relación 1:1, se puso en operación el sistema soxhlet a reflujo por 2 horas a 70oC. Los extractos fueron sometidos a un proceso de concentración en un equipo rotavapor marca Heidolph por alrededor de 2 horas a una temperatura de $60^{\circ} \mathrm{C}$ y 20 rpm por 3 horas aproximadamente. Los concentrados se enfriaron a temperatura ambiente, se depositaron en frasco ámbar con tapa y fueron refrigerados para los ensayos posteriores.

Para obtener el extracto del fruto, el procedimiento fue el siguiente:

\section{Extracto Metanólico de Fruto de $\boldsymbol{S}$. samán}

Al cartucho que contiene la muestra molida se le adicionó $200 \mathrm{~mL}$ de alcohol metanol, se puso en operación el sistema soxhlet a reflujo por 2 horas a $70 \circ C$. A continuación, este extracto se sometió a un proceso de concentración en un equipo rotavapor marca Heidolph por alrededor de 2 horas a una temperatura de $600 \mathrm{C}$ y $20 \mathrm{rpm}$ por 2 horas aproximadamente y al final del proceso, el concentrado fue enfriado a temperatura ambiente, depositado en frasco ámbar con tapa y refrigerado para los ensayos posteriores.

\section{Tamizaje Fitoquímico de los Extractos}

A los tres extractos se les aplico un ensayo de tamizaje fitoquímico; de acuerdo con los métodos descritos por Evans et $a l^{20}$ y Harborne ${ }^{21}$, con el fin de determinar cualitativamente la presencia o ausencia de metabolitos secundarios de interés antagónico frente a hongos. Estos análisis fueron: Test para Alcaloides:Test de Dragendorff's, Test de Mayer,Test de Wagner,Test del ácido pícrico (1\%), Test para Flavonoides:Test del
Amonio y Test del Cloruro de Aluminio,Test para Glicósidos, Test para Esteroides y Terpenoides, Test para Saponinas:Test de Emulsión,Test del froting, Test para Taninos:Test del Subacetato de plomo,Test del cloruro Férrico, Test para compuestos ácidos, Test para resinas:Test del precipitado y Test del color.

\section{Elaboración de Biopelícula}

Para el presente trabajo se usó una biopelícula a base de almidón de yuca y gelatina de bovino, que cuenta con excelentes propiedades mecánicas de dureza, resistencia y extensibilidad; así como, acción de barrera semipermeable a la humedad y al oxígeno, propuesta por Acosta Dávila ${ }^{22}$.

La biopelícula propuesta está estructurada por tres compuestos: Polímero: Solución de gelatina de origen bovino (2\% S.T.) y Almidón de yuca (2\% S.T.) en una relación 50:50. Plastificante: Glicerol (0,5\% del total de la mezcla) y Solvente: Agua destilada.

\section{Activación del hongo Colletotrichum gloeosporioides}

Para las pruebas antagónicas de los extractos de corteza y fruto de S. samán, se usó una cepa certificado por el Centro de Investigación Biotecnológica del Ecuador (CIBE) del hongo Colletotrichum gloesporioides spp., con código MCIBE-H148.

Las muestras recibidas del hongo $\mathrm{C}$. gloesporiodes fueron activadas tomando una muestra visible del hongo y pre enriqueciéndolas en un caldo de cultivo BHI (Brain Heart Infussion Broth) de "Titan Biotech Limited ${ }^{\circledR}$ ", para luego ser inoculadas en agar inclinado PDA (Potato Dextrosa Agar) de "DIFCO ${ }^{\circledR}$ " por 5 días a $24 \pm 20 \mathrm{C}$. Los parámetros tomados en cuenta para este proceso son temperatura de incubación: 24£2oC, tiempo de incubación: 5-7 días y aislado de la luz.

Determinación de la concentración mínima inhibitoria
(CMI) de los extractos crudos de corteza y fruto de
Samanea samán frente a cultivos puros de Colletotrichum gloeosporioides in vitro

Para la determinación de la CMI, se ensayaron en primera instancia varias concentraciones de los tres tipos de extractos analizados (200, 175, 150, 125, 100, 50, 25, $\left.12.5 \mathrm{mgL}^{-1}\right) \mathrm{me}-$ diante el uso de placas multipozos ( 96 pozos) adicionando en cada uno de estos $100 \mathrm{mgL}^{-1}$ de agar PDA, $20 \mathrm{mgL}^{-1}$ del hongo y las diferentes concentraciones a ensayar en un numero de cuatro replicas por concentración ensayada. Estas placas fueron incubadas a $24 \pm 20 \mathrm{C}$ por 48 horas en incubadora.

Una vez determinado el tipo de extracto y las concentraciones con los mayores efectos inhibitorios, se sometieron estas concentraciones $\left(90,100,110,120\right.$ y $\left.130 \mathrm{mgL}^{-1}\right)$ del extracto metanólico (EM) a un nuevo análisis a través del uso de placas multipozos ( 96 pozos) usando la mismas dosificaciones y condiciones de incubación que en el proceso previo, en un numero de diez replicas por concentración ensayada.

Con la identificación de las concentraciones con mayores perfiles inhibitorios $\left(120,130\right.$ y $\left.140 \mathrm{mgL}^{-1}\right)$ del extracto metanólico (EM), se procedió a realizar el ensayo para la determinación de la concentración mínima inhibitoria (CMI) a través de la técnica del pozo en placa con siembra por estrías.

Cada placa con $20 \mathrm{~mL}$ de agar PDA fueron inoculadas con cultivo fresco del hongo $\mathrm{C}$. gloeosporioides mediante la técnica de estrías con asa, posteriormente se procedió a realizar un pozo en el centro de la placa mediante el uso del segmento ancho de una punta de micropipeta de $9 \mathrm{~mm}$ de radio y $5 \mathrm{~mm}$ de profundidad aproximadamente. En estos pozos fueron depositados las concentraciones a ensayar por cuadruplicado (120,130 y $140 \mathrm{mgL}^{-1}$ ) del extracto metanólico (EM). Se usaron testigos del crecimiento del hongo mediante la incubación de 
un blanco (agar PDA), Control (agar+hongo) y Solvente (agar+metanol) en concentraciones de $60 \mathrm{mgL}^{-1}$ y $120 \mathrm{mgL}^{-1}$ para el solvente, estas placas fueron incubadas por 7 días a $24 \pm 20 \mathrm{C}$.

La metodología para la recolección de los datos para la determinación de la concentración mínima inhibitoria (CMI) del extracto metanólico (EM); a través de la medición de los halos de inhibición, fueron tomados del trabajo de (Awerbuch \& Lustman, 1987) ${ }^{23}$. Los datos fueron registrados y se determinó las medias de los halos de inhibición con su respectivo error estándar para cada lectura; así también, con estos datos procesados se construyó una curva de respuesta inhibitoria para determinar los IC10, IC50 e IC90.

Determinación del efecto antagónico del sistema Biopelicula: Extracto de Samanea samán frente a cultivos puros de Colletotrichum gloeosporioides in vitro:

Con la identificación de la CMI del extracto metanólico (EM) en la sección anterior, se procedió a formular un sistema de Biopelicula:Extracto $(1: 0,14)$ respectivamente, considerando los tiempos de agitación para la homogenización del sistema por 1 min y 5 min respectivamente.

Se procedió a tomar cada placa con $20 \mathrm{~mL}$ de agar PDA y a inocularlas con cultivo fresco del hongo C. gloeosporioides mediante la técnica de estrías con asa, posteriormente se procedió a realizar un pozo en el centro de la placa mediante el uso del segmento ancho de una punta de micropipeta de $9 \mathrm{~mm}$ de radio y $5 \mathrm{~mm}$ de profundidad aproximadamente. A las placas inoculadas se les adiciono $1 \mathrm{~mL}$ del sistema Biopelicula:Extracto, cada ensayo se realizó por quintuplicado y para los dos tiempos de homogenización planteados, estas placas fueron incubadas por 7 días a $24 \pm 20 \mathrm{C}$.

Con los resultados obtenidos del crecimiento micelial, se procedió a realizar un ensayo adicional aumentando la concentración del sistema Biopelicula:Extracto $(1: 0,2)$ con un tiempo de agitación de 5 minutos y los resultados fueron recogidos, enlistados y analizados en la siguiente sección.

\section{Resultados y discusión}

\section{Tamizaje Fitoquímico}

Con los ensayos completados del tamizaje fitoquímico se observó que en el extracto metanólico (EM) del fruto hubo presencia de alcaloides, glucósidos, esteroides, terpenoides, saponinas, taninos y resinas; mientras que hubo ausencia de flavonoides y compuestos acídicos que coinciden con los resultados obtenidos por Obasi et al. ${ }^{13}$. Los resultados también mostraron que para los solventes más polares del estudio el extracto etanòlico (EE) de la corteza mostró únicamente presencia de esteroides, terpenoides y saponinas; así como, para el extracto hidroetanólico (EHE) también de la matriz corteza, solamente tuvo presencia de esteroides, saponinas y resinas, difiriendo de los resultados expuestos por Milian-Dominguez et $a L^{7}$ exceptuando solo para los contenidos de saponinas y resinas.

Estos resultados nos brindaron una valiosa visión de las características de cada matriz y solvente usado para la obtención de los diferentes extractos, encontrando prometedores resultados para el extracto metanólico del fruto del S. saman respecto de su contenido de metabolitos secundarios; específicamente hablando de los alcaloides, saponinas y taninos; estos últimos comúnmente conocidos como acido tánico y reportados por su elevado potencial antimicrobiano y anti fúngico según Naveen-Prasad R. et al. ${ }^{11}$. Tabla 1.

Al completar los ensayos para la determinación de la concentración mínima inhibitoria (CMI) para los extractos puros; luego de un análisis estadístico, se encontró que la dosis de 140uL del extracto metanólico (EM) presento la media más alta de inhibición para el hongo estudiado (C. gloeosporioides); este resultado difiere completamente de los datos presentados por Obasi N. y Col. (2010)13 quien no encontró efectos inhibitorios significativos de los extractos metanólicos del fruto frente a las cepas de hongos estudiadas a concentraciones de $10 \mathrm{mgmL}^{-1}$ a $100 \mathrm{mgmL}^{-1}$. Por otro lado, los resultados obteni-

\begin{tabular}{|l|c|c|c|}
\hline ENSAYO PARA METABOLITOS SECUNDARIOS & EE & EHE & EM \\
\hline ALCALOIDES & & & \\
\hline Test de Draggendorf's & - & - & + \\
\hline Test de Mayer & - & - & + \\
\hline Test de Wagner & - & - & + \\
\hline Test del ácido picrico & & - & + \\
\hline FLAVONOIDES & - & - & - \\
\hline Test del amonio & - & - & - \\
\hline Test del cloruro de Aluminio & - & - & + \\
\hline GLICOSIDOS & + & + & + \\
\hline ESTEROIDES & + & - & + \\
\hline TERPENOIDES & & & \\
\hline SAPONINAS & + & + & + \\
\hline Test de emulsión & + & + & + \\
\hline Test del froting & & & \\
\hline TANINOS & - & - & + \\
\hline Test del subacetato de plomo & - & - & + \\
\hline Test del cloruro férrico & - & - & - \\
\hline COMPUESTOS ACIDOS & & & + \\
\hline RESINAS & - & + & + \\
\hline Test del precipitado & - & + & + \\
\hline Test del color & & \\
\hline
\end{tabular}

Tabla 1. Resultados experimentales tamizaje fitoquímico.EE(Extracto Etanòlico 96\%) Corteza S. saman).EHE (Extracto Hidroetanólico (50:50) Corteza S. saman). EM (Extracto Metanólico Fruto S. saman). Presencia (+), Ausencia (-). 
dos en este trabajo se presentan con ventaja frente a los resultados expuestos por Aguilar-Alonso et al. ${ }^{24}$ quienes reportaron un $\mathrm{CMI}$ para extractos etanòlicos de ruda y pirúl de $150 \mathrm{mgL}^{-1}$ y $300 \mathrm{mgL}^{-1}$ respectivamente, mientras que Avila-Sosa et al. ${ }^{4}$ reportaron valores de $\mathrm{CMI}$ del orden de $400 \mathrm{mgL}-1$ en extractos etanòlicos de orégano mexicano, jarilla y pericón, por lo que la concentración identificada del EM presento ventajas in vitro competitivas en comparación con los trabajos en referencia para ser usada como el agente biocída en la biopelícula (Tabla 2).

\begin{tabular}{|c|c|c|}
\hline & \multicolumn{2}{|c|}{ CMI } \\
\hline EM $(\boldsymbol{\mu} \mathbf{L})$ & Rtox & \multicolumn{1}{c|}{ Rmut } \\
\hline $\mathbf{1 2 0}$ & $4,075 \pm 0,2986$ & $4,875 \pm 0,2394$ \\
\hline $\mathbf{1 3 0}$ & $5,625 \pm 0,4787$ & $6,375 \pm 0,2394$ \\
\hline $\mathbf{1 4 0}$ & $7,625 \pm 0,4787$ & $8,75 \pm 0,1443$ \\
\hline
\end{tabular}

Tabla 2. Resultados de la media y error estándar de los halos de inhibición para los extractos metanólicos (EM) puros. rtox (radio de la zona de toxicidad), rmut (radio de la zona de mutagenocidad), CMI (Concentración mínima Inhibitoria).

Así también, con las lecturas de inhibición se construyó una curva de respuesta con la que se encontró para este extracto crudo (EM) un IC10 $=61,05 \mu \mathrm{L}, \mathrm{IC} 50=81,8557 \mu \mathrm{L}$ y IC90=107,99 $\mu \mathrm{L}$. (Tabla 3).

\begin{tabular}{|c|c|c|}
\hline \multicolumn{3}{|c|}{ EM $(\mathbf{2 0 0 ,} \mathbf{1 0 0 , 5 0 ,} \mathbf{5 5 , 1 2 . 5 )} \boldsymbol{\mu L}$} \\
\hline IC10 & IC50 & IC90 \\
\hline $\mathbf{6 1 , 0 5}$ & 81,8557 & 107,99 \\
\hline
\end{tabular}

Tabla 3. Valores de respuesta inhibitoria para los extractos metanólicos (EM) puros.

La curva de supervivencia (Figura 1) nos permite obtener la función específica con la que se puede determinar el porcentaje de supervivencia (IC) para este extracto puro.
En la evaluación del efecto antagónico de los sistemas biopelícula: extracto, se determinó que para las dosis de $140 \mu \mathrm{L} /$ $\mathrm{mL}$, el factor de agitación influye tácitamente en la respuesta inhibitoria frente al hongo en estudio. Al aumentar el tiempo de agitación de 1 minuto a 5 minutos, la respuesta antagónica a esta concentración fue mayor llevando el crecimiento micelial a un nivel de reproducción medio, lo que esto presenta un descubrimiento innovador para este tipo de investigaciones, donde sus análisis se han concentrado en los efectos inhibitorios generados por el tipo de sustancia antimicrobiana y su concentración, sin tomar en cuenta el efecto de la difusión de los extractos en el polímero, como un parámetro promotor de la función biocída de los sistemas. (Tabla 4).

Al aumentar la concentración del extracto en el sistema a $200 \mu \mathrm{L} / \mathrm{mL}$, se observó una respuesta inhibitoria considerablemente mayor, consiguiendo un nivel de reproducción in vitro bajo para el hongo C. gloesporiodes responsable de la antracnosis en mango (Mangifera indica), estos resultados tienen un comportamiento más estable respecto de los valores reportados por Ribeiro-Goncalves et al. ${ }^{25}$ quienes presentaron datos bastantes dispersos de inhibición in vitro que van desde $15 \mathrm{~mL}^{-1}$ como la concentración de mayor inhibición, hasta $6000 \mathrm{~mL}^{-1}$ para la concentración de menor efecto en extractos comerciales naturales.

Por otro lado, trabajos presentados por Maqbool M. et al. ${ }^{26}$ encontraron efectos fungicidas en sistemas goma arábiga (GA) al $10 \%$ y aceite esencial de limoncillo (LG) y canela (CM) al 0,05\% y 0,4\% respectivamente, lo que nos da una relación 1:0,08 y 1:0,01 para cada sistema y Bosquez-Molina E. et al. ${ }^{27}$ reportaron niveles de reducción de la incidencia de C. gloesporioides del $\mathbf{1 0 0 \%}$ en sistemas goma mezquite al $5 \%$ y aceite esencial de tomillo $(0,1 \%)$ y Lima mexicana $(0,5 \%)$ que en relación con el presente trabajo tendríamos una formulación 1:0,02 y 1:0,01 polímero extracto lo que nos indica claramente que las características de la biopelícula inciden directamente sobre la acción fungicida de los extractos. Para todas las dosis ensayadas en el análisis de la respuesta antagónica del sistema, se

$$
F i c=\frac{-0,0182+(1,0115-(-0,0182))}{1+\left(\frac{x}{E C 50}\right)^{-(-7,5360)}}
$$

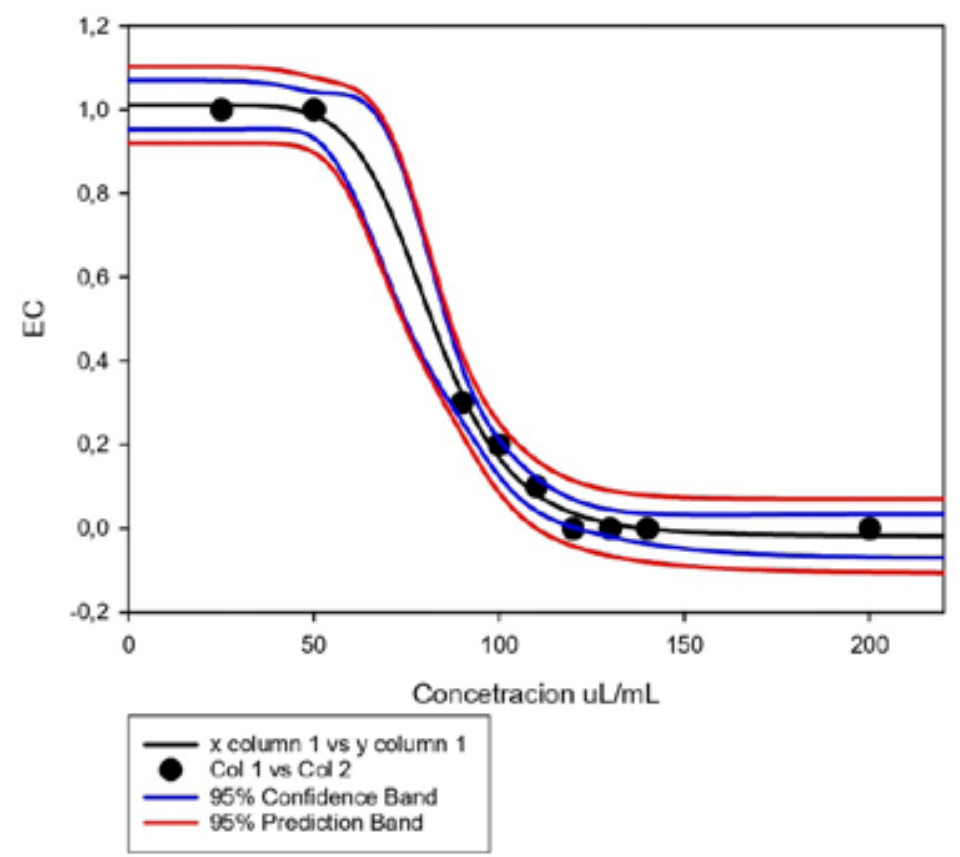

Figura 1. Curva de respuesta inhibitoria del extracto metanólico (EM) puro. 


\begin{tabular}{|l|c|c|c|c|c|}
\hline & \multirow{2}{*}{ Placa } & \multirow{2}{*}{$\begin{array}{c}\text { Extracto } \\
(\boldsymbol{\mu} \mathbf{L} / \mathbf{m L})\end{array}$} & $\begin{array}{c}\text { Agitación } \\
(\mathbf{m i n})\end{array}$ & \multicolumn{3}{|c|}{ Crecimiento Micelial } \\
\cline { 5 - 6 } & & Bajo & Medio & Alto \\
\hline BioP 1 & 140 & 1 & & & +++ \\
\hline BioP 2 & 140 & 1 & & & +++ \\
\hline BioP 3 & 140 & 1 & & & +++ \\
\hline BioP 4 & 140 & 1 & & & +++ \\
\hline BioP 5 & 140 & 1 & & & +++ \\
\hline BioP 1 & 140 & 5 & & ++ & \\
\hline BioP 2 & 140 & 5 & ++ & & \\
\hline BioP 3 & 140 & 5 & + & & \\
\hline BioP 4 & 140 & 5 & & + & \\
\hline BioP 5 & 140 & 5 & & + & \\
\hline BioP 1 & 200 & 5 & +++ & & \\
\hline BioP 2 & 200 & 5 & +++ & & \\
\hline BioP 3 & 200 & 5 & +++ & & \\
\hline BioP 4 & 200 & 5 & +++ & & \\
\hline BioP 5 & 200 & 5 & +++ & & \\
\hline
\end{tabular}

Tabla 4. Resultados del efecto antagónico de los sistemas Biopelicula:Extracto (EM) de Samanea samán frente a C. gloeosporiodes.

encontró que las placas después del día cinco de incubación presentaban un crecimiento de colonias negras con una taxonomía parecida al género Aspergillus, lo que ratifica la premisa propuesta por Lopez-Malo A. et al. ${ }^{28}$ de que el crecimiento radial de las cepas de hongos están directamente relacionadas no solo con la concentración del agente antimicrobiano y el tipo de polímero como dispersante; sino también, la actividad de agua (Aw) y el pH como factores fundamentales para una óptima funcionalidad del sistema.

\section{Conclusiones}

De manera general, conocemos que los metabolitos secundarios de mayor interés antimicrobianos están identificados como alcaloides, taninos y saponinas; y están estos justamente presentes de mejor manera, en el extracto metanólico (EM) del fruto del Samanea samán, por lo que el estudio identifica claramente a la matriz y al solvente como los medios para la mejor extracción de estos compuestos. Así también, el presente trabajo introdujo al tiempo de agitación para la difusión del extracto en la biopelícula como un parámetro innovador en términos investigativos, ya que influye directamente sobre la respuesta antagónica de estos sistemas.

Finalmente, el estudio nos muestra que la composición del polímero también influye directamente en la respuesta inhibitoria del extracto en el sistema; y promueve la aparición de microorganismos competitivos, presumiblemente por una modificación de las condiciones de $\mathrm{pH}$ y la actividad de agua (Aw) de la biopelícula originalmente.

\section{Referencias bibliográficas}

1. Arauz, Luis Felipe. Mango Anthracnose: Economic Impact and Current Options For Integrated Managaement. Plant Disease.2000. 84(6), 600-611. https://doi.org/10.1094/PDIS.2000.84.6.6002.

2. Valdés, L. A., Calero Consuegra, D. L., Gómez, A., Carballo, M. E., Capote, M., González, I., ... Rohde, W. Caracterización morfológica, cultural y patogénica de aislados de Colletotrichum sp. produciendo antracnosis en mango (mangifera indica L.). La Granja, (2017). 26(2), 38. https://doi.org/10.17163/lgr.n26.2017.04
3. Funes, M. J. B., \& Salazar, J. E. C. (s. f.). Factores que inciden en la innovación tecnológica y productiva del sector del mango en el Ecuador. 13.

4. Avila-Sosa, R., \& Lopez-Malo, A. Aplicacion de sustancias antimcrobianas a peliculas y recubrimientos comestibles. Temas Selectos de Ingenieria en Alimentos, (2008). 2, 10.

5. Vázquez-Briones, M. C., \& Guerrero-Beltran, J. A. Recubrimientos de frutas con biopelículas. (2013). 10.

6. Delgado, D. C., Hera, R., \& Cairo, J. (s. f.). Samanea saman, árbol multipropósito con potencialidades como alimento alternativo para animales de interés productivo. 9.

7. Milián Domínguez, J. C., Monroy, O. I., \& Valdés, H. (s. f.). Caracterización fitoquímica de Samanea Saman (Jacq.) Merr. (Algarrobo). (2017). 5, 13

8. Gutierrez Martinez, P., Bautista Baños, S., \& Barrera Necha, L. Uso potencial de extractos vegetales, aceites esenciales y quitosano para reducir el daño causado por hongos postcosecha en productos hortofrutícolas. Avances en ciencia y tecnología alimentaria en México,(2013).1(1).Disponible en : https://www.researchgate. net/publication/272623331

9. Delgado, D. C., Hera, R., \& Cairo, J. (s. f.). Samanea saman, árbol multipropósito con potencialidades como alimento alternativo para animales de interés productivo.9.

10.lqbal Azhar, M., Mohtasheemul, H., \& Farah Mazhar, A. Some biological evaluations on Samanea saman. Pakistan Journal of Pharmocology, (2009). 26(1), 47-53.

11. Prasad, R. N., Viswanathan, S., Devi, J. R., Nayak, V., Swetha, V. C., Parathasarathy, N., \& Rajkumar, J. (s. f.). Preliminary phytochemical screening and antimicrobial activity of Samanea saman. 3.

12. Aguilar López, J., Jaén Jiménez, J. C., Vargas Abarca, A. S., Jiménez Bonilla, P., Vega Guzmán, I., Herrera Núñez, J., ... Soto Fallas, R. M. Extracción y evaluación de taninos condensados a partir de la corteza de once especies maderables de Costa Rica. Revista Tecnología en Marcha, (2012). 25(4), 15. https://doi. org/10.18845/tm.v25i4.615

13. Obasi Nnamdi, L., Egbounu Anthony, C. C., Ukoha Pius, O., \& Ejikeme Paul, M. Comparative phytochemical and antimicrobial screening of some solvent extracts of Samanea saman (fabaceae or mimosa) pods. African Journal of Pure and Applied Chemistry, (2010). 4, 206-212.

14. Vázquez-Briones, M. C., \& Guerrero-Beltran, J. A. Recubrimientos de frutas con biopelículas. (2013). 10.

15. Bartz, D. J. A., \& Brecht, Dr. J. K. (s. f.). Reporte Final sobre potenciales modificaciones en el empacado de mangopara mejorar el control postcosecha de antracnosis. 10 
16. Brecht, J. K. Manual de Practicas para el Mejor Manejo Postcosecha del Mango. National Mango Borad. (2009).

17. Tajkarimi, M. M., Ibrahim, S. A., \& Cliver, D. O. Antimicrobial herb and spice compounds in food. Food Control, (2010). 21(9), 11991218. https://doi.org/10.1016/j.foodcont.2010.02.003

18. Stella-Ramirez, L., \& Marín, D. (2009). Metodologías para evaluar in vitro la actividad antibacteriana de compuestos de origen vegetal. Xv, 42, 263-268

19. Thippeswamy, S., Praveen, P., Mohana, D.C., \& Manjunath, K. (2011). Antimicrobial Evaluation and phytochemical analysis of a known medicinal plant Samanea saman (Jacq.) Merr. Against some human and plant pathogenic bacteria and fungi. International Journal of Pharma and Bio Sciences, 2(2).

20. Evans, W. C., Evans, D., \& Trease, G. E. (2009). Trease and Evans pharmacognosy (16th ed). Saunders/Elsevier.

21. Harborne, J. B. Phytochemical methods: A guide to modern techniques of plant analysis (1998). (3rd ed). Chapman and Hall.

22. Acosta Davila, S. Propiedades de films de almidon de yuca y gelatina. Incorporacion de aceites esenciales con efecto antifungico. Universidad Politecnica de Valencia. (2014).

23. Awerbuch, T. E., \& Lustman, L. A mathematical model for determining minimal inhibitory concentrations (MICs) via diffusion assays. Journal of Theoretical Biology, (1987). 129(2), 219-230. https://doi.org/10.1016/S0022-5193(87)80014-0
24. Aguilar-Alonso, P. Navarro-Cruz, A. R. Sánchez-Flores, A. B., Ávila-Sosa, R., \& Meneses-Sánchez, M. D. la C. Efecto antifúngico de extractos de plantas originarias del estado de Puebla sobre Colletotrichum gloeosporioides. CienciaUAT, (2013). 7(2), 06. https://doi.org/10.29059/cienciauat.v7i2.17

25. Ribeiro, J. G., Serra, I. M. R. de S., Araújo, M. U. P., Ribeiro, J. G., Serra, I. M. R. de S., \& Araújo, M. U. P. Uso de produtos naturais no controle de antracnose causado por Colletotrichum gloeosporioides em mamão. Summa Phytopathologica, (2016). 42(2), 160164. https://doi.org/10.1590/0100-5405/2023

26. Maqbool, M. Postharvest application of gum arabic and essential oils for controlling anthracnose and quality of banana and papaya during cold storage. Postharvest Biology and Technology, (2011). 6.

27. Bosquez-Molina, E., Jes Ãos, E. R., \& Bautista-BaÃ, S.. Inhibitory effect of essential oils against Colletotrichum gloeosporioides and Rhizopus stolonifer in stored papaya fruit and their possible application in coatings. Postharvest Biology and Technology, (2010).6

28. Lopez-Malo, A., Alzamora, S. M., \& Palou, E. Aspergillus flavus growth in the presence of chemical preservatives and naturally occurring antimicrobial compounds. International Journal of Food Microbiology, (2005).10.

Received: 22 noviembre 2020

Accepted: 7 enero 2021 